Our preliminary findings obtained in these patients indicate a possibility of determining the location of the coagulation electrode and suggest the need for extension of such observations.

\title{
5. Experimental and Clinical Studies on the Destruction Method at the Stereotaxic Surgery
}

\author{
Junichi Wakisaka, Shinken Kuramoto, Mitsuo Watanabe, \\ and Yoshiichi OHNAKA \\ Ist Department of Surgery, Kurume University, School of Medicine
}

On laboratory cats with electrodes permanently implanted in the cerebrum the Oil-Wax infusion aimed at the pallidum as well as the destruction by means of high frequency current aimed at the thalamus was conducted stereotaxically. Changes occurring in the destroyed site and in its surroundings on that occasion were followed with the lapse of time in both aspects of local impedance of the brain and the depth EEG, and after sacrificing the animals observation was made macroscopically and histologically on the areas concerned. On the other hand, with the human brain local impedance fluctuations as well as changes of the depth EEG caused by destruction with high frequency current were also investigated.

Moreover, on the basis of the data obtained from the cases of parkinsonism to which the Oil-Wax infusion or the destruction with high frequency current aimed at th same target point was applied comparison was made between the former and the latter as a destruction method at stereotaxic surgery respectively.

The present comparative study revealed that in both cases of the Oil-Wax infusion and the destruction with high frequency current changes in the impedance fluctuation were remarkable, but those in the EEG were not so evident. Although changes in the impedance fluctuation due to Oil-Wax infusion were recognizable for a while after infusion, it gradually returned to the state before infusion. On histological examination it was found that this finding was advancing gradually. On the contrary, as regards to changes in the impedance fluctuation due to destruciton with high frequency current the impedance fluctuation returned, in the peripheral part, to the state before destruction in the course of time gradually, whereas it did not appear even 3 weeks after destruction. From the histological point of view it proved that there existed an irreversible destruction in the central part.

So far as the above-mentioned cases of parkinsonism to which either the OilWax infusion or the destruction with high frequency current respectively aimed at the same target point were concerned, it was ascertained that although in any 
cases examined marked effects could be demonstrated for a while from just after operation, recurrence took place in the former and, in contrast, in the latter almost any recurrence did not occur. From these results it can be concluded that the method of destruction by means of high frequency current represents an ideal one to destroy the brain tissues for stereotaxic surgery as compared with the method of Oil-Wax infusion.

\title{
6. Ultrasonic Examination of Intracerebral Destructive Lesion in Stereotaxic Surgery
}

\section{- The Third Report-}

\author{
Akira Nishimoto, Kazuhiko Sadamoto, Shin Ueda, \\ Kiyoshi IwatsukI and Yukifumi Honjo \\ Department of Neurological Surgery, Okayama University Medical School
}

Stereotaxic operations for the extrapyramidal disorders or intractable pains are one of the choice of the surgery. There have been carried out many methods of destruction of the target currently. But it was impossible to observe the condition of the destructed lesion itself directly during and after surgery. The lesion has been controled by the experience of the surgeon, by the clinical symptoms and by the experimental data.

Aloka SSD-10 universal ultrasonic medical analyser, MSU compound sector manual unit were used under the aid of monitor of Aloka SSD-2 A-scope apparatus, and transducer with barium titanate of $2.25 \mathrm{Mc} / \mathrm{sec}$. The ultrasonic sector scanning was performed usually on the operative side vertically and horizontally. The probe was placed just above the ear mantle and directed to the target of the thalamus. The lesion echo was obtained about 1.3 to $1.5 \mathrm{~cm}$. lateral to the midline echo in cases of the destruction of the ventrolateral nucleus of the thalamus. In the case that the lesion was placed in the centromedian, the echo was located adjacent to the midline echo with 0.3 to $0.7 \mathrm{~cm}$. lateral to it.

When the echograms magnified twice, the lesion echo was drawn as two layers in some cases. It can be considered that these echoes were formed by the reflection from the lateral and medial wall of the lesion or were made by artifact due to the complicated acoustic structure of the lesion itself. The reflection from the lesion with compound scope was noted in 49 out of 54 cases. The follow-up observation of the lesion echo was performed in all cases. Even after 5 months, the lesion echo was readily noted in all cases. The echo was located with a distance from the midline echo. The distance was proved to be exactly same length roentgenologically, although the size and shape of the lesion echo 